Théologiques

Théologiques

\title{
L'esprit dans quelques textes magistériaux : point de vue exégétique
}

\section{André Myre}

Volume 2, numéro 2, octobre 1994

L'esprit

URI : https://id.erudit.org/iderudit/602407ar

DOI : https://doi.org/10.7202/602407ar

Aller au sommaire du numéro

\section{Éditeur(s)}

Faculté de théologie de l'Université de Montréal

\section{ISSN}

1188-7109 (imprimé)

1492-1413 (numérique)

Découvrir la revue

Citer cet article

Myre, A. (1994). L'esprit dans quelques textes magistériaux : point de vue exégétique. Théologiques, 2(2), 63-71. https://doi.org/10.7202/602407ar
Résumé de l'article

Dans la Bible, « Esprit » est un concept utilisé pour exprimer l'origine divine d'une expérience. L'article qui suit recherche les fins que poursuivent trois documents récents de l'Église (le Catéchisme de l'Église catholique, l'encyclique Veritatis splendor et le document de la Commission biblique pontificale intitulé : L'interprétation de la Bible dans l'Église), quand ils parlent de l'Esprit. Ces textes sont analysés quant à leur sens de l'histoire, leur acceptation des acquis de l'exégèse et leur niveau d'actualisation. L'étude conduit à la conclusion que "Esprit ", dans ces documents, n'est pas utilisé pour rendre compte d'une expérience mais pour faire admettre un pouvoir. 


\title{
L'esprit dans quelques textes magistériaux: point de vue exégétique
}

\author{
André MYRE \\ Faculté de théologie \\ Université de Montréal
}

\section{RÉSUMÉ}

Dans la Bible, «Esprit * est un concept utilisé pour exprimer l'origine divine d'une expérience. L'article qui suit recherche les fins que poursuivent trois documents récents de l'Église (le Catéchisme de l'Église catholique, l'encyclique Veritatis splendor et le document de la Commission biblique pontificale intitulé : L'interprétation de la Bible dans l'Église), quand ils parlent de l'Esprit. Ces textes sont analysés quant à leur sens de l'histoire, leur acceptation des acquis de l'exégèse et leur niveau d'actualisation. L'étude conduit à la conclusion que «Esprit *, dans ces documents, n'est pas utilisé pour rendre compte d'une expérience mais pour faire admettre un pouvoir.

Le concept occidental d'esprit a deux racines 1 . La vie l'a certes fait grandir mais il ne serait pas le grand arbre qu'il est devenu sans ses racines. Et ses racines ne sont pas mortes, puisque lui vit. Mais il est des branches menacées de sclérose, parce que se coupant de la vie souterraine. De cela il sera ici question.

1 Comme il est ici question de l'utilisation du concept en Occident, le vocable «esprit» sera utilisé. Mais pour rester fidèle aux deux racines du concept, il faudrait parler du «souffle-esprit». "Souffle» rendant bien le dynamisme de la racine sémitique, * esprit » se situant du côté gréco-romain (même si le mot, dans son origine latine de * spiritus », avait autant le sens d'air en mouvement que la rouach hébraïque ou le pneuma grec). 
Un simple coup d'œil au mot esprit, dans Le petit Robert, révèle la richesse du concept. On passe de la première acception, souffle de Dieu, à émanation des corps, à être immatériel, à réalité pensante, à aptitude intellectuelle, à attitude ou idée orientant l'action. Dans les pages qui suivent, on se concentrera sur la première. C'est déjà chose osée.

Le sous-titre qui coiffe ces lignes en indique le point de vue, celui d'un bibliste. C'est une perspective originale sur la réalité, qui mérite d'être décrite. J'ai abordé mon sujet plein de bonne volonté et ce n'est pas sans une certaine excitation intellectuelle que j'ai accepté d'étudier le rôle attribué à l'Esprit dans quelques témoins de la production magistérielle actuelle, en vue de la situer par rapport aux acquis récents de l'exégèse ${ }^{2}$.

J'ai tenté de me donner une grille d'interprétation relativement simple. Voici donc les principaux points que j'ai voulu vérifier:

1. Sens de l'histoire. Il importe de savoir si les écrits étudiés font justice aux périodes historiques traversées par l'Église, et aux différentes inculturations que la foi chrétienne y a connues.

2. Acceptation des acquis de l'exégèse. Le mot "Esprit ", qui est probablement toujours une force impersonnelle dans la Bible ${ }^{3}$, tire son sens des contextes dans lesquels il est utilisé et des auteurs qui l'emploient. Il n'a pas le même sens partout ${ }^{4}$. De plus, l'utilisation du concept vise moins à parler de l'Esprit en soi qu'à pointer du doigt des réalités qui ou bien sortent de l'ordinaire ou bien ne s'expliquent que par un recours à la Puissance d'intervention de Dieu. Il sera intéressant de vérifier si les patients efforts des exégètes pour se retrouver dans ce dédale de sens ont fini par porter fruit.

3. Sens de l'actualisation. La foi chrétienne n'existe qu'actualisée dans une culture. Il en a toujours été ainsi et c'est vrai même des énoncés du Nouveau Testament. On ne connaît ce qui provoque l'actualisation qu'à travers une actualisation déjà réalisée. Et aucune ne peut prétendre rendre

2 Le champ d'investigation est limité, mais la petite grille de lecture offerte plus bas pourrait être utilisée avec profit pour dégager les conceptions de l'Esprit dont témoignent la production théologique, les communautés ecclésiales de base, les groupes populaires, etc. Cet élargissement des horizons serait nécessaire en vue d'un discours pertinent sur l'Esprit tel qu'il est compris aujourd'hui.

3 Cetre prise de position est discutée, mais est exégétiquement défendable. En tout cas, elle rend compte de la plupart des textes bibliques (sinon tous).

$4 \quad$ L'article d'O. Mainville, dans le présent numéro de Théologiques, en fait foi. 
compte de cet inconnu-en-soi une fois pour toutes ou en être la formulation définitive qui n'aurait plus qu'à être retraduite en d'autres langages. Il faudra voir où on en est rendu dans l'effort de conceptualisation de cette force d'intervention que les anciens nommaient "Esprit ".

Trois textes récents pourvoiront aux fins de notre enquête: le Catéchisme de l'Église catholique (CÉC), l'encyclique Veritatis splendor (VS) et le document de la Commission biblique pontificale intitulé: L'interprétation de la Bible dans l'Église (IBÉ) ${ }^{5}$. L'auteur de l'encyclique renvoyant au catéchisme comme à un " texte de référence sûr et authentique " (VS §5), je commence par le catéchisme, suivant l'ordre chronologique de parution des textes.

\section{Le Catéchisme de l'Église catholique}

Faire une synthèse des énoncés du catéchisme sur l'Esprit servirait peu mon propos, je me contente de commenter quelques caractéristiques qui m'apparaissent significatives par rapport aux critères qui viennent d'être formulés.

Disons d'entrée de jeu que ce n'est pas en lisant le catéchisme qu'on pourra se mettre au fait des acquis de l'exégèse. Non seulement aucun exégète n'y est jamais cité, mais on n'y fait aucune lecture exégétique des textes. On ignore l'exégèse, quand on ne la contredit pas, tout simplement. Quelques exemples suffiront.

Ainsi, pour le catéchisme, "Croire en l'Esprit Saint c'est donc professer que l'Esprit Saint est l'une des Personnes de la Trinité Sainte,... " (CÉC $\S 685)$. Une telle affirmation discrédite la foi néotestamentaire, car Paul et Luc croyaient certainement en l'Esprit Saint, mais ne l'ont jamais envisagé comme personne. Pour eux, il était la puissance impersonnelle de Dieu, confiée au Christ dans l'exaltation. On ne peut s'exprimer comme le fait le catéchisme que si on n'a pas le sens des périodes historiques traversées par l'Église et des diverses inculturations que la foi y a connues. On part des expressions patristiques et classiques de la foi, et on les projette

5 Catéchisme de l'Église catholique, Ottawa, CÉCC, 1993; Karol Wojtyla (JeanPaul II), Lettre encyclique Veritatis Splendor, Cité du Vatican, Libreria editrice vaticana, 1993; Commission biblique pontificale, «L'interprétation de la Bible dans l'Église », dans La documentation catholique (2 janvier 1994) 13-44. Comme le souligne le cardinal J. Ratzinger, dans sa préface, la Commission biblique pontificale n'est pas un organe du magistère mais elle a son appui. Ce n'est donc pas trop forcer les choses que d'inclure son texte dans cette étude. 
en arrière dans le Nouveau Testament ou en avant vers aujourd'hui, comme si elles avaient toujours existé ou étaient définitives. Les recherches exégétiques et historiques sont ainsi totalement ignorées.

Un second exemple. On lit encore dans le catéchisme: «La Parole de Dieu et son Souffle sont à l'origine de l'être et de la vie de toute créature: au Saint Esprit il convient...d'animer la création... " (CÉC §703). S'il est vrai que dans les premières traditions vétérotestamentaires l'Esprit est à l'œuvre dans la création, les mentions de cet agir diminuent progressivement. Dans les derniers livres de l'Ancien Testament l'Esprit se concentre sur les personnalités humaines et, jamais, dans le Nouveau, ne le présentet-on comme agissant dans la création non humaine, ni même dans les humains qui se situent en dehors de la foi chrétienne. Cette caractéristique du Nouveau Testament n'aurait-elle pas dû inquiéter les auteurs du catéchisme? Comment comprendre que ceux-ci fassent rejaillir la personnalisation de l'Esprit sur l'ensemble de l'histoire de la foi, et, par ailleurs, méconnaissent la concentration de l'Esprit scripturaire sur la personne humaine? Sans doute, en partie du moins, par un refus de faire intervenir une lecture exégétique des textes. Mais pourquoi ce refus, qui s'exprime dans une interprétation toujours non critique des textes ${ }^{6}$ ? Pourquoi inviter les exégètes au travail (CÉC \$115-119), si c'est ensuite pour ignorer les résultats de leurs recherches?

La réponse se trouve sans doute dans un passage important, situé au début du document, sur la révélation (CÉC \$76-79). On y parle de la transmission de l'Évangile par les apôtres qui, oralement d'abord, ont rapporté "ce qu'ils tenaient des suggestions du Saint-Esprit ", puis, par écrit, "sous l'inspiration du même Esprit Saint ». Ceux-ci laissèrent comme successeurs les évêques, auxquels ils "transmirent leur propre charge d'enseignement", transmission qui sera ininterrompue "jusqu'à la consommation des temps". Cette transmission vivante s'accomplit "dans l'Esprit Saint", de sorte qu'ainsi les croyants sont introduits «dans la vérité tout entière ".

Il y aurait de longs débats à faire sur cette lecture traditionnelle des débuts ${ }^{7}$. Exégétiquement et historiquement parlant, elle ne va pas de soi.

6 Voir en particulier les pages sur l'Esprit par rapport à Jésus et l'Église (CÉC 717-741).

7 On trouvera une lecture exégétique de la transmission des ministères dans A. MYRE, “Nouveau Testament et ministères», dans Des ministères nouveaux? Une question qui se pose (en collab.), Montréal, Paulines; Paris, Médiaspaul, 1985, p. 95. 162. 
Mais l'important, en ce qui concerne le but de la présente étude, n'est pas là; il se situe plutôt dans le rôle qui est attribué à l'Esprit dès le début $d u$ document. Et ce rôle est clair, c'est celui de justifier le pouvoir d'interprétation du magistère, qui s'exprimera dans le reste du catéchisme. On comprend donc, dès les premières pages, pourquoi le document ne se soucie pas d'exégèse. Il n'en a pas besoin. Car le même Esprit qui a inspiré les apôtres, inspire les auteurs du document et les fait accéder à la vérité tout entière. Ils n'ont donc pas à s'attarder à de fastidieuses études. L'Esprit les inspire et c'est en se laissant guider par eux que les exégètes - obligés, eux, à un délicat travail qui les met face à une multitude de sens possibles - en arriveront à la "juste interprétation " des textes (CÉC \$116). Les exégètes ont à lire le magistère, s'ils veulent bien comprendre l'Écriture, mais le magistère n'a pas à connaître l'exégèse en vue de bien interpréter cette même Écriture. Voilà pourquoi on retrouve dans le catéchisme une lecture non historique, non exégétique et non actualisée (au sens strict) de l'Écriture. Interprétation faussée de la part d'un exégète frustré? Jetons un coup d'œil sur Veritatis splendor.

\section{L'encyclique Veritatis splendor}

Je me permets de commencer par présenter une assez longue citation, car je la crois particulièrement éclairante.

Données par Dieu dans l'Ancienne Alliance et parvenues à leur perfection dans la Nouvelle et éternelle alliance, en la personne même du Fils de Dieu fait homme, les prescriptions morales doivent être fidelement conservées et actualisées en permanence dans les différentes cultures tout au long de l'histoire. La charge de leur interprétation a été confiée par Jésus aux Apôtres et à leurs successeurs, assistés spécialement par l'Esprit de vérité. ... Dans la catéchese morale des Apôtres, parallèlement aux exhortations et aux indications relatives au contexte historique et culturel, se trouve un enseignement éthique avec des normes précises de comportement. Cela apparaît aussi dans leurs Lettres, qui contiennent l'interprétation, guidée par l'Esprit Saint, des préceptes du Seigneur à vivre dans les différentes situations culturelles... (VS \$25-26)

Certains des principaux "successeurs" dont parle ce texte sont nommés la première fois que l'encyclique mentionne l'Esprit: il s'agit des Souverains Pontifes qui, "personnellement ou avec le Collège épiscopal, ... avec la certitude de l'assistance de l'Esprit de vérité " se sont prononcés sur les exigences morales qui doivent influencer les aspects les plus 
importants de la vie humaine (VS §4). C'est à ce "seul magistère " qu'il a été confié d'interpréter la parole de Dieu (VS \$27). Interviennent alors

ceux qui enseignent la théologie morale... Ils ont le grave devoir d'instruire les fidèles ... au sujet de tous les commandements et de toutes les normes pratiques que l'Église énonce avec autorité (VS \$110).

Les fidèles, interpellés par cette vérité qui leur est ainsi interprétée et cette Loi qui se découvre à eux, n'ont plus qu'à obéir librement, menés en cela par l'Esprit.

On le voit, le rôle de l'Esprit est solidement en place. Quand on pose la question: "A quelle expérience répond l'utilisation du concept d'Esprit dans l'encyclique?", la réponse est aisée. À l'expérience du pouvoir. "Esprit " est un concept utilisé par le magistère pour justifier le pouvoir qu'il se reconnaît d'exprimer des vérités d'ordre intellectuel ou éthique, en ne laissant aux autres croyants d'autre choix que celui d'y adhérer librement.

L'encyclique ne voit l'histoire que comme le milieu où se fait la transmission de vérités immuables mais actualisables. Si l'Esprit agit au cœur des théologiens, exégètes ou croyants, c'est pour leur faire admettre l'authenticité des déclarations magistérielles. Mais aucune découverte, expérience ou actualisation digne de ce nom ne se fait à leur niveau. Car il se trouve, dans la tradition chrétienne interprétée par le magistère, de par la volonté de Dieu, "un enseignement éthique avec des normes précises de comportement » qui a à se vivre dans les différentes cultures. Pour l'encyclique, le croyant ou la croyante n'a pas à se laisser conduire par l'Esprit, mais bien par la Loi (interprétée par le magistère) à laquelle l'Esprit les mène:

... celui qui se laisse "mener par l'Esprit » $(\mathrm{G} a 5,16)$ et désire servir les autres trouve dans la Loi de Dieu la voie fondamentale et nécessaire pour pratiquer l'amour librement choisi et vécu (VS $\$ 18)^{8}$.

D'après cette façon de voir, aucune actualisation ou inculturation vraie n'est possible car des formulations définitives existent, dogmatiques ou morales, dont on n'a qu'à juger comment les rendre compréhensibles ou vivables dans les différentes situations culturelles. Il n'y a pas d'inconnuen-soi, de dynamisme orienté qui provoque à de radicales inculturations. On le comprend. Si tel était le cas, si la vérité était à faire plutôt qu'à

8 On comprend que l'encyclique cite $\mathrm{Ga}$ 5, 16 mais pas 5,18: * Mais si l'Esprit vous anime vous n'êtes plus sous la Loi.» 
dire, à découvrir dans le faire avant que de se dire, ce ne serait plus le magistère qui détiendrait le pouvoir dans l'Église. Mais l'Esprit, présent au çeur de tout croyant, de toute croyante. Et c'est lui qui déterminerait le visage concret de l'Église, dans chaque culture, à travers l'histoire. Et le rôle des pasteurs serait d'être un appel permanent au discernement de l'Esprit à l'œuvre dans l'Église. Au nom de l'Esprit dont elle se réclame, l'encyclique ferme la porte à l'Esprit à l'œuvre dans l'Église.

\section{L'interprétation de la Bible dans l'Église}

Le document de la Commission biblique pontificale parle relativement peu de l'Esprit, mais ce qu'il en dit (ou n'en dit pas) mérite d'être relevé.

Jadis l'Esprit a guidé les auteurs bibliques et les a inspirés, même si ces derniers n'étaient pas conscients de tous les niveaux de sens de leurs textes (IBÉ pp. 30,33,34). C'est lui qui a fait discerner le canon des Écritures (IBÉ p. 33). A travers les âges, les évêques sont éclairés par l'Esprit pour guider les croyants qui, eux-mêmes, ont l'Esprit (IBÉ pp. $28,30,33,35,4243)^{9}$. De plus, "L'Église compte sur des exégètes animés par le même Esprit qui a inspiré l'Écriture ", y compris un " nombre croissant de femmes exégetes $»$, pour nourrir le peuple de Dieu. Mais, en dernier recours, c'est le magistère qui est chargé d'exprimer la véritable interprétation de l'Écriture: "il consulte à cet effet des théologiens, des exégètes et d'autres experts, dont il reconnaît la légitime liberté " (IBÉ p. 35).

Le code Esprit joue le même rôle dans le document que dans les autres textes étudiés auparavant, mais la mention de la consultation même si elle n'est soumise à aucune règle et tarde à produire des fruits - mérite d'être soulignée. Le texte reconnaît implicitement que l'Esprit n'apprend pas tout au magistère.

La dernière partie du document, sur "L'interprétation de la Bible dans la vie de l'Église ", devrait susciter de bonnes discussions. On y écrit, entre autres choses, que l'Église accueille la Bible comme "Parole de Dieu qui s'adresse à elle, et au monde entier, dans le temps présent "; les textes bibliques ont une portée telle qu' « il est nécessaire d'appliquer leur

9 De façon surprenante, le document reconnaît l'Esprit aux évêques, aux croyants individuels et aux exégètes, mais ne l'attribue pas expressément aux prêtres et diacres (IBÉ p. 35). 
message aux circonstances présentes et de l'exprimer dans un langage adapté à l'époque actuelle» (IBÉ p. 39). On dira même :

Le fondement théologique de l'inculturation est la conviction de foi que la Parole de Dieu transcende les cultures dans lesquelles elle a été exprimée et a la capacité de se propager dans les autres cultures, de façon à atteindre toutes les personnes humaines dans le contexte culturel où elles vivent (IBÉ p. 40).

Ce qu'il y a de remarquable dans ces deux citations, c'est que l'Esprit n'y est pas mentionné. Là même où il jouerait éminemment un des rôles majeurs que la Bible lui attribue, on omet d'en parler. Et ce n'est pas simple oubli. C'est qu'en parler troublerait l'harmonie du code magistériel. En effet, il est essentiel pour rester dans la logique du discours ecclésial officiel ${ }^{10}$, que la parole de Dieu soit un texte, un message codifié, un donné connu et immuable, qu'on peut propager dans les autres cultures. Il est essentiel également que le rôle de l'Esprit soit restreint à renvoyer vers ce discours connu pour le faire interpréter. Car s'il fallait que la Bible ne soit pas le message, mais qu'une expression du message, expression située, inculturée, relative, d'un inconnu-en-soi, toujours derrière le texte, jamais mis de l'avant, toujours dynamisant mais jamais capturé, alors tout l'édifice construit sur le code croulerait ${ }^{11}$.

Pourtant, grandes sont les chances que tel soit bien l'Esprit chrétien, ce souffle dont on ne sait ni l'origine ni la destination (Jn 3,8), qui ne se reconnaît qu'une fois qu'on s'est laissé mener par lui. Cet absolu, le seul de notre expérience, face auquel tout dans l'Église n'est que relatif. Cet Esprit qui dynamise à édifier des constructions, constructions qu'il s'empresse de miner à la base quand elles rendent impossible l'expérience du souffle.

L'exégète n'est pas celui qui peut dire où souffle cet Esprit aujourd'hui. Mais son expérience du passé peut lui permettre d'indiquer dans quelle direction on a le plus de chances de le trouver.

Les textes étudiés sont d'une cohérence remarquable, qui n'est possible que parce qu'il s'agit de constructions intellectuelles qui n'ont pas

10 Et la Commission biblique pontificale l'exprime pour une large part, même si elle ne fait pas partie du magistère.

11 Le document de la Commission est traversé par un souffle historique évident et est au fait de la situation actuelle de l'exégèse. Mais, quand il s'agit d'interprétation, d'herméneutique et d'actualisation, les perspectives qui seraient naturelles à l'exégèse sont mises de côté au profit du code institutionnel sur l'Esprit. 
à s'ajuster à la complexité du réel et de la vie ${ }^{12}$. Le recours à l'Esprit, contrairement au discours biblique, n'est pas nécessité par une expérience dont on ne saurait rendre compte autrement, mais par le besoin d'une autorité transcendante pour faire admettre un pouvoir qui, sans elle, aurait peine à se justifier. Un tel Esprit sert de caution à un ensemble de constructions d'ordre juridique, éthique, dogmatique ou institutionnelle, qui n'ont pas à subir le test du réel, puisque c'est le réel qui doit s'ajuster à elles.

Pendant ce temps, ceux qu'on a encouragés à penser leur foi à partir des sciences humaines, à l'inculturer pour faire advenir l'inconnu-en-soi dans le concret de la matière et de l'humain, en lien étroit avec la vie de leur peuple, sont rendus dans un ailleurs auquel le magistère, à cause de son idéologie, se refuse d'accéder. Triste sort d'un magistère qui se coupe de son peuple. Et pour qui la réalité dynamique de l'Esprit est la pire menace au pouvoir qu'il a érigé sur lui.

12 Dans le cas du document de la Commission biblique, cette remarque vaut pour les lignes qui portent sur les liens avec le magistère, mais pas pour le reste du texte, attentif aux difficultés du métier d'exégète. 